\title{
The new Shinkansen rescheduling system for drivers and crew
}

\author{
H. Shimizu ${ }^{1}$, H. Tanabe ${ }^{2}$, S. Honda ${ }^{2} \&$ K. Yasura ${ }^{2}$ \\ ${ }^{1}$ JR East Japan Information Systems Company, Japan \\ ${ }^{2}$ East Japan Railway Company, Japan
}

\begin{abstract}
We are now undertaking development of the new rescheduling plan system for drivers and crew, to synchronize with the forecast train plan. The East Japan Railway Company has five Bullet Train (Shinkansen) lines operating about 800 trains per day, dispatching nearly 250 drivers and 350 crew per day to make the trains operational. To keep the operation plan and management of Shinkansen in order, we have the system named COSMOS (Computerized Safety Maintenance and Operation systems of Shinkansen). The drivers and crew work plans are made with the drivers and crew rescheduling system, one of the subsystems of the COSMOS transportation planning system. Each driver and crew works on their train according to the work plan normally decided on beforehand. However, sometimes trains would be unable to operate on schedule when weather is bad or car troubles occur. In such cases, the work plan must be redesigned. Should the change affect two or more crews' the redesigned plan should only change the plan of one crew member. It is very difficult to make this plan change because the delay of the train changes minute by minute. To change the crews' work plans adequately, we have developed a new system. This system displays the contradictions of the change plans of the crew if the train delay happens. Work planners make the change plan to solve these contradictions.

The changing of jobs can be selected for the most suitable options, accurately and quickly, based on the computer supporting systems. We reduce the delay time of trains by utilizing this system and it will improve our services for customers.
\end{abstract}

Keywords: train scheduling, driver scheduling, crew scheduling, forecast, realtime rescheduling. 


\section{Introduction}

The East Japan Railway Company has the latest computer system that holds the plan and the management of an elaborate train schedule of SHINKANSEN. This system enables a safe and accurate train operation.

This system is called "Computerized Safety Maintenance and Operation Systems for SHINKANSEN" - "COSMOS". COSMOS has eight sub-systems the "Transportation plan system" called "TPS", the "Operation control system" called "OCS", the Railway yard work management system, the Maintenance works control system", the Rolling stock control system", the Facility control system", the Facility monitoring system" and the "Electric power control system". In COSMOS, these eight sub-systems are cooperating elaborately.

We have already been using COSMOS for 11 years since 1995 . We are now advancing the redevelopment of TPS and OCS. It aims at a further function improvement, and the development of the new feature that uses a new technology - for example, the Train rescheduling system using constraint programming, etc. - is included in this development.

In this thesis, "Rescheduling for driver and crew, using forecast train scheduling data" of the new features is described.

\subsection{About forecast train scheduling data}

In the OCS, the forecast train scheduling data is made with the system so far. This data is a calculation of the scheduled departure and arrival times at each train station and the forecasted arrival and departure times according to actual events. The forecast train scheduling data, called "FTS", periodically displays the information once a minute in this system. When the train is delayed, this system calculates the delay time of the departure and arrival at each station.

\subsection{About rescheduling for drivers and crew}

TPS includes two scheduling systems for drivers and crew. The first one is a function to perform planning and management of the driver and crew scheduling several months in advance. Another function, as driver and crew scheduling is planned beforehand, reschedules when the train is delayed. This function is called "Rescheduling for driver and crew". Hereafter, it is called "RDC".

\section{Comparison and new feature with current functions}

\subsection{Current problems}

The current RDC has some problems. In this paragraph, current problems are enumerated and each solution for them is described in the next system.

There are the following problems.

(a) The plan of drivers and crew cannot be rescheduled to synchronize with the delay of trains. 
(b) The plan of drivers and crew cannot be rescheduled to synchronize with the rescheduling of trains.

(c) If no adjustment happens, within a short time the current system fails.

We introduce the case, (b) that can actually happen as follows.

There is a plan that the driver changes from train " 1 " to train " 2 " at station A. Figure 1 shows this. At this time, using the track of station "A", the train is changed from track " 2 " to " 1 " in the train plan as shown in Figure 2.

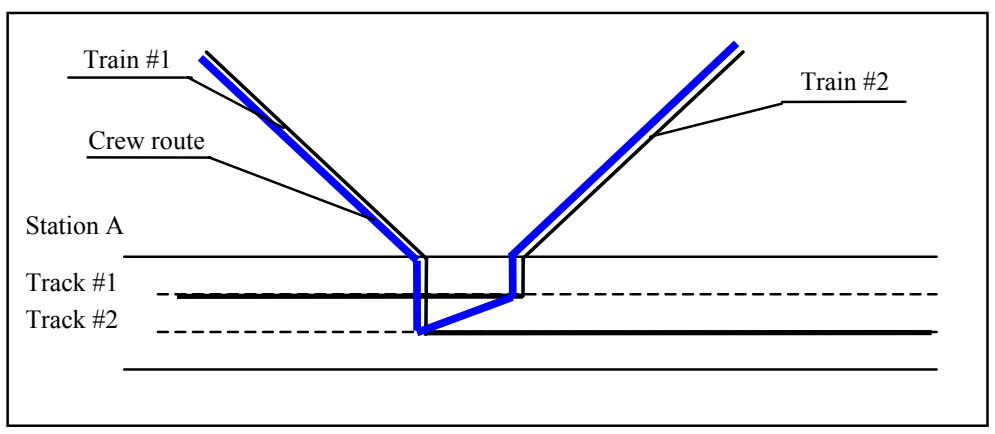

Figure 1: $\quad$ Before track changing.

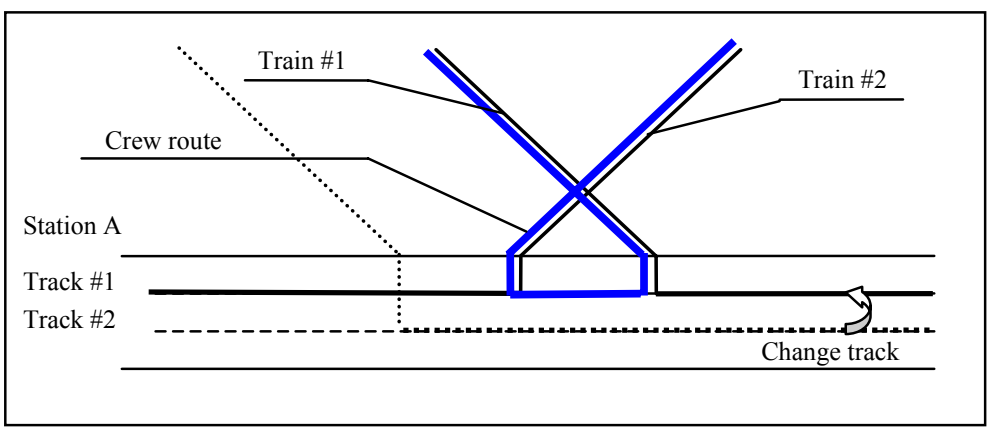

Figure 2: After track changing.

As a result, no adjustment is generated in the train plan and the driver plan.

In a word, train " 1 " cannot arrive at station " $A$ " if train " 2 " doesn't leave.

On the other hand, if train 1 doesn't arrive, train 2 cannot depart, because the same driver works on both trains.

In the current system, such an event might not turn out satisfactorily.

The plan has a big influence as the train is delayed when such an event happens. 
In the new system, the mechanism that no adjustment can be understood at the early stage is necessary to prevent such an event beforehand.

\subsection{For the problem solution}

We assumed that we added the following function to new RDC.

(a') FTS is reflected in real time to RDC.

(b') The plan change of trains is reflected in real time to RDC.

(c') Enables us to understand no adjustment of the plan that happens previously in several hours.

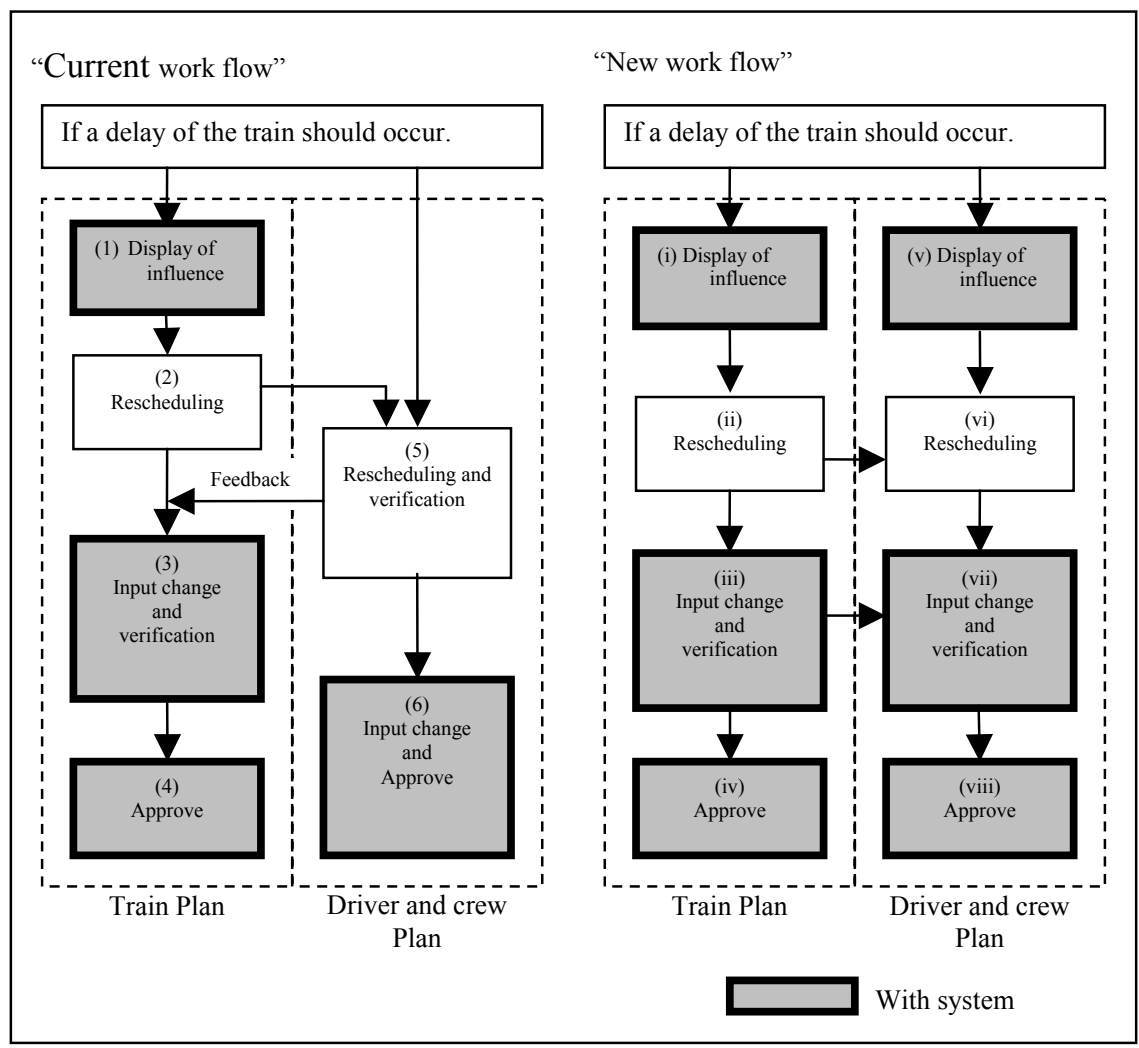

Figure 3: $\quad$ Current and new work flow.

Figure 3 shows current and new workflow for drivers and crew rescheduling. (a') corresponds to (v) of Figure 3. It is shown to be able to display the delay of the train influences on planning with the system.

(b') corresponds to (vii). A current system takes the change in the train rescheduling plan like (5) outside the system. A new system takes the change like (vii) inside the system. 
(c') is achieved as a whole by cooperate with the train rescheduling and the drivers and crew rescheduling.

\section{New functions of RDC}

This chapter describes the new feature of RDC.

\subsection{Reflection of FTS in RDC}

Each minute or every instance when the plan is changed, the system makes FTS. Each time, this FTS is stored in the database. RDC periodically gets FTS from the database. FTS is reflected in the diagram screen and the horizontal bar screen in RDC.

Figure 4 shows the example of displaying the screen when the train is delayed.

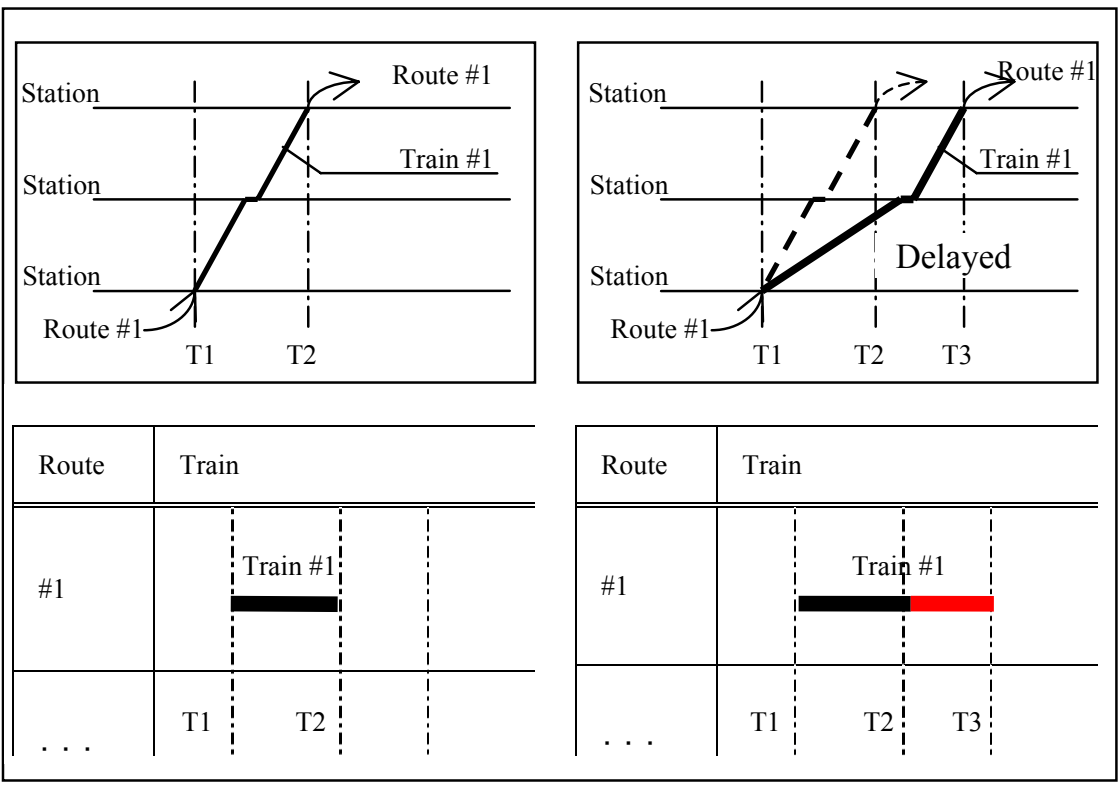

Figure 4: $\quad$ Example of displaying the screen in RDC.

\subsection{Display of change recommendation point of plan by FTS in RDC}

This chapter explains three functions of RDC. It is the one to display both of these three functions plan change recommendation points.

\subsubsection{Attention of change of drivers and crew plan}

This attention means that the driver or crew will be late for the train that works next, because the train on which they are working is delayed. In this case, the attention mark is displayed on the screen. 
There is a plan that a certain driver or crew works with train "1" and train "2". He changes it from train "1" to train "2" at station "C". At this time, the condition of displaying this attention mark is shown by the following expressions.

$$
\mathrm{T}_{\mathrm{a}}(1, \mathrm{C})+\mathrm{N}(\mathrm{C})>\mathrm{T}_{\mathrm{d}}(2, \text { C) --------- formulate } 1
$$

$T_{d}(r, s)$ : Forecast departure time

$\mathrm{T}_{\mathrm{a}}(\mathrm{r}, \mathrm{s})$ : Forecast arrival time

$\mathrm{N}(\mathrm{s})$ : The change time required

R: Train number

S: Station

Figure 5 shows the example of displaying the screen.

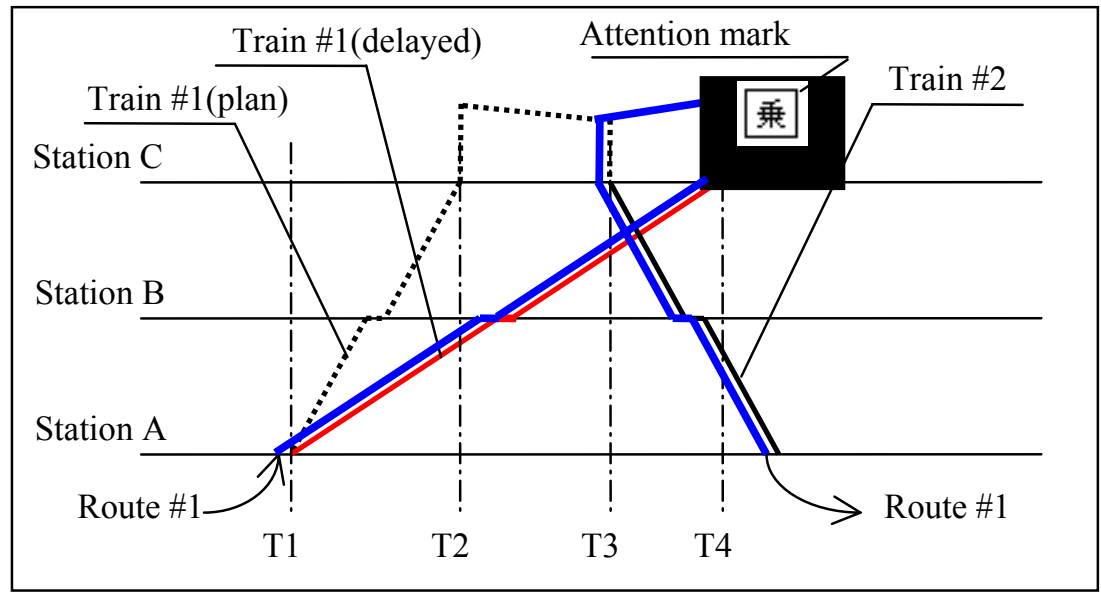

Figure 5: Attention mark of change of drivers and crew plan.

\subsubsection{Attention of the train assigned no driver or crew}

When one train is newly added in the train rescheduling, driver or crew has not been allotted this new train in RDC yet.

At this time, on the screen, it is displayed that the color of the line of this new train is different from usual. Moreover, the round mark painted out in white is displayed at the first station of the section that not is even if the driver or crew allocates it. Figure 6 shows this.

\subsubsection{Attention of difference between station getting on and getting off}

When one train becomes driving discontinuance among trains by which a driver or crew is scheduled to work, the station that he gets off with the train ahead of that and the station gets on with the train afterwards are different.

At this time, the attention mark is displayed on the screen. And a line is displayed between the station of getting off and the station of getting on. 
In Figure 3, it is shown that the station where driver or crew gets off train "1" and the station where he takes train " 3 " are different by discontinuance of train " 2 ".

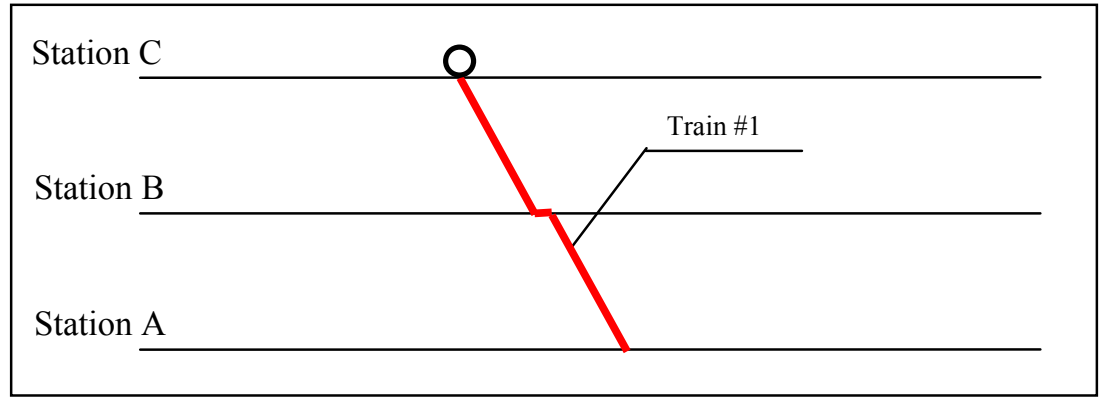

Figure 6: Attention of the train assigned no driver or crew.

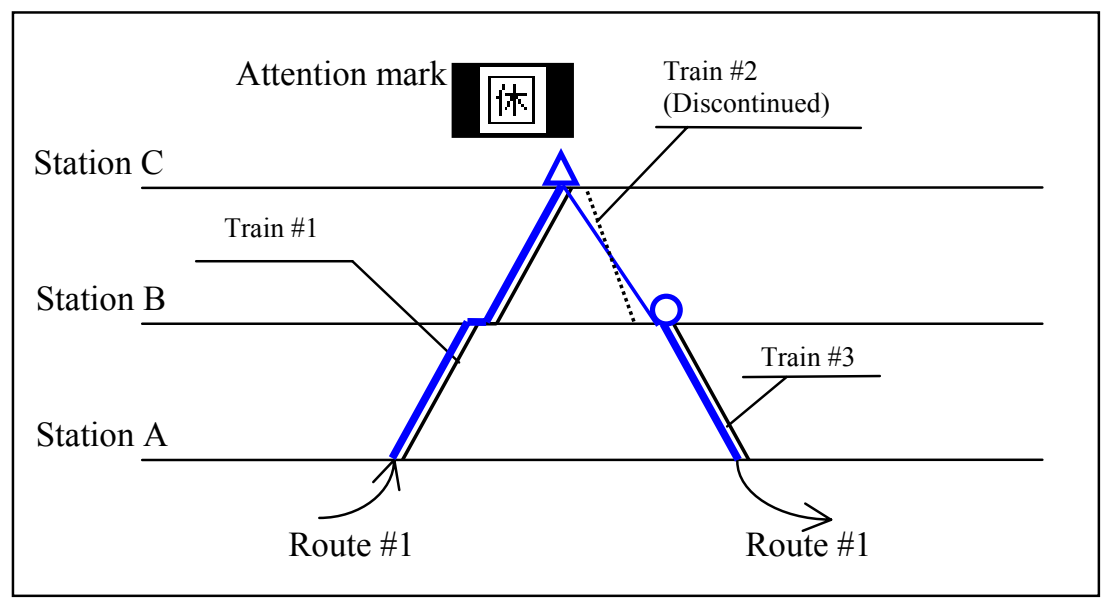

Figure 7: Attention of difference between station getting on and getting off.

\section{Conclusions}

In this thesis, the cooperation of FTS and RDC has been described. We think that RDC becomes more strategic and more efficient by cooperating with FTS.

In the railway transportation, RDC is very important. The Shinkansen transportation doesn't work out even if there is a correspondence only in the train plan. It works out with a correspondence in both the train plan and the drivers and crew plan.

We can understand no adjustment of the plan that happens previously in several hours with new RDC.

For example, we can understand for several hours or more though we were able to understand driver's or crew's lack only immediately before the current. 
234 Computers in Railways X

Then, we can beforehand let a preliminary driver go to the departure station of the train allocated nobody, and can let him get on the train.

From the above-mentioned, we think that RDC becomes more flexible now, and the Shinkansen transportation becomes stabler. 